A - preparing concepts

$\mathrm{B}$ - formulating methods

$\mathrm{C}$ - conducting research

$\mathrm{D}$ - processing results

E - interpretation and conclusions

$\mathrm{F}$ - editing the final version

Received: 2020-07-27

Accepted: 2020-09-16

Published: 2020-09-19

\section{A comparative study of shoulder muscle strength, sense of proprioception and internal/external rotation flexibility between adolescent athletes with and without scapular asymmetry}

\author{
Bihter Akınoğlu*1,2,A-F (D), Banu Kabak ${ }^{2, B, D, F}$ (D), Aydin Balci ${ }^{3, C-D, F}$ (D), \\ Tuğba Kocahan ${ }^{2, A-B, D-F}$ (D), Adnan Hasanoğlu' $2, B, D-F$ (iD \\ ${ }^{1}$ Department of Physiotherapy and Rehabilitation, Faculty of Health \\ Sciences, Ankara Yıldırım Beyazıt University, Ankara, Turkey \\ ${ }^{2}$ The Ministry of Youth and Sports, Sports General Directorship, Department \\ of Health Services, Center of Athlete Training and Health Research, Turkey \\ ${ }^{3}$ Ankara Yıldırım Beyazıt University, Yenimahalle Training and Research \\ Hospital, Sports Medicine Department, Turkey
}

*Correspondence: Bihter Akınoğlu; Department of Physiotherapy and Rehabilitation, Faculty of Health Sciences, Ankara Yıldırım Beyazıt University, Ankara, Turkey; email: rgkardelen@yahoo.com

\begin{abstract}
Introduction: Scapular asymmetry may affect the biomechanics of the shoulder girdle joints and muscles by changing the contraction angles of the muscles. The purpose of this prospective cohort, matched-controlled study was to compare shoulder muscle strength, proprioception sense and internal/external rotation flexibility between adolescent athletes with and without scapular asymmetry.

Material and methods: Nineteen athletes of tennis, fencing, shooting, archery, gymnastics, and badminton branches with left side (non-dominant) scapular asymmetry were included in the study as asymmetry group. Nineteen athletes who have similar gender, sports branch, professional experience, physical characteristics with asymmetry group were included in the study as the control group. Scapular asymmetry was measured using a tape measure with the Lateral Scapular Slide Test. Shoulder horizontal abduction/adduction isokinetic muscle strength and shoulder abduction/adduction isometric muscle strength was tested by an isokinetic dynamometer. The proprioception sense of the glenohumeral joint was evaluated with a digital inclinometer, and the flexibility of the internal and external rotation of the shoulder was evaluated by the flexibility test.

Results: There was not any significant difference between the groups in isokinetic muscle strength, isometric muscle strength, and proprioception sense of shoulder $(\mathrm{p}>0.05)$. Shoulder internal rotation flexibility of both dominant and nondominant sides was higher in the asymmetry group than the control group $(\mathrm{p}<0.05)$.

Conclusions: It was determined that the flexibility of shoulder internal rotation in adolescent athletes with scapular asymmetry was higher than those without asymmetry.
\end{abstract}

Keywords: posture, sport, scapula

\section{Introduction}

Providing balanced scapular stabilization facilitates muscular function by controlling the length-tension relationship of the scapular muscles [1]. It is stated that the scapular asymmetry, which is defined as the difference in the amount of scapula diverging from the midline of the back, is observed especially in athletes performing asymmetrical sports such as baseball and tennis, and it usually occurs due to the traction of the muscles and

This is an Open Access journal, all articles are distributed under the terms of the Creative Commons Attribution-NonCommercial-ShareAlike 4.0 International (CC BY-NC-SA 4.0). License (http://creativecommons. org/licenses/by-nc-sa/4.0/). 
ligaments of the dominant side [2,3]. Researchers state that scapular asymmetry is due to the weakness of the scapulothoracic muscles; while on the other hand, some others state that it is caused by the strength imbalance of the scapular position muscles [3-5].

The mobility and stabilization of the shoulder region depends on the functional integrity and organization of the musculoskeletal system [6-8]. Considering the shoulder's biomechanics, a difference in the movement of any of the joints composing the shoulder girdle hinders the normal upper limb movements and causes abnormal scapulohumeral rhythm [6-8]. Scapular asymmetry lead to biomechanical abnormalities and change periscapular muscle activity [8]. This can affect the position of joint and proprioception $[9,10]$. This situation leads to impaired functional integrity and organization of the shoulder joint, and it may cause various shoulder problems [6-8]. There is not any clear evidence about the relationship between scapular asymmetry and shoulder injuries and the causes of the possible relationship. It is stated that in the presence of scapular asymmetry; altered muscle strength, flexibility, and abnormal scapular rhythm precipitate the development of subacromial impingement and glenohumeral instability. Therefore, scapular asymmetry may be associated with shoulder injuries $[3,6,11,12]$.

Scapular movement was asymmetrical in patients with shoulder pain [7] and changed periscapular muscle activity in the presence of scapular asymmetry might cause shoulder injuries [8]. Scapular asymmetry shows the imbalance of the muscles around the scapula [13], and shoulder pain is associated with shoulder horizontal abduction and internal rotation angles [14]. Shoulder pain is associated with some factors affecting mobility and stability of the shoulder, such as proprioception, muscle strength, and flexibility $[8,14,15]$. The relationship between shoulder pain and scapula has been investigated in the athletes too, and shoulder pain has been shown to be associated with the abnormal scapular position [14]. The importance of determining and preventing factors that cause shoulder pain and injury is indisputable in terms of athletes' health and sports performance.

To the authors' knowledge, there is not any study examining muscle strength, sense of proprioception, and shoulder range of motion in the presence of scapular asymmetry. However; the literature reveals the hypothesis that scapular motion and position changes may affect muscle strength, proprioceptive feedback, and joint range of motion. For this reason, the aim of the study is to examine the shoulder joint muscle strength, sense of proprioception, and internal/external rotation flexibility in adolescent athletes with scapular asymmetry and compare them with adolescents athletes without asymmetry.

\section{Materials and method}

Thirty-eight adolescents athletes (tennis $=4$, fencing $=4$, shooting $=4$, archery $=6$, gymnastics $=18$, badminton $=2$ ) participated in this research design conducted in the training and research center of athletes. According to Kibler's Lateral Scapular Slide Test (LSST), 19 athletes with $1.5 \mathrm{~cm}$ or above scapular asymmetry [16] on the non-dominant left side were included in the study group. Nineteen adolescent athletes with the same age / gender / sport branch / sport year as the participants with scapular asymmetry and without asymmetry $(<1 \mathrm{~cm})$ according to LSST were selected as the control group. It was determined that there was a significant difference between the groups in the scapular asymmetry measurements (Tab. 1).

Tab. 1. Comparison of demographic characteristics, professional experience and scapular asymmetry of the groups

\begin{tabular}{|c|c|c|c|c|}
\hline & $\begin{array}{l}\text { Control group } \\
(\mathrm{n}=19)\end{array}$ & $\begin{array}{l}\text { Asymmetry group } \\
\qquad(\mathrm{n}=19)\end{array}$ & $z^{\mathfrak{E}}, t^{¥}$ & $\mathrm{p}$ \\
\hline Age (year) & $15.89 \pm 1.76$ & $15.63 \pm 2.17$ & $-0.687^{\mathfrak{k}}$ & $0.492^{\mathfrak{E}}$ \\
\hline Weight (kg) & $54.16 \pm 10.23$ & $56.44 \pm 12.94$ & $-0.602^{¥}$ & $0.551^{\#}$ \\
\hline Height (m) & $1.64 \pm 0.11$ & $1.64 \pm 0.10$ & $0.241^{¥}$ & $0.811^{¥}$ \\
\hline BMI $\left(\mathrm{kg} / \mathrm{m}^{2}\right)$ & $19.86 \pm 2.17$ & $20.82 \pm 3.14$ & $-1.106^{¥}$ & $0.276^{¥}$ \\
\hline Professional Experience (year) & $7.05 \pm 2.91$ & $7.71 \pm 3.11$ & $-0.517^{\mathfrak{k}}$ & $0.605^{\mathfrak{1}}$ \\
\hline Scapular Difference in Neutral Position (cm) & $0.39 \pm 0.42$ & $1.46 \pm 0.70$ & $-4.312^{\mathfrak{1}}$ & $<0.001^{£}$ \\
\hline Scapular Difference in $45^{\circ}$ Abduction Position (cm) & $0.53 \pm 0.38$ & $1.21 \pm 0.74$ & $-2.996^{£}$ & $0.003^{£}$ \\
\hline Scapular Distance in $90^{\circ}$ Abduction Position $(\mathrm{cm})$ & $0.39 \pm 0.39$ & $1.14 \pm 1.04$ & $-2.365^{\mathfrak{1}}$ & $0.018^{£}$ \\
\hline
\end{tabular}

p: comparison of study and control group $\left({ }^{£}\right.$ Mann Whitney U Test, ${ }^{¥}$ Independent Samples T Test). 
Right limb dominancy, at least 3-year professional experience, and volunteering to participate in the study were the inclusion criteria for the study. Lower limb length difference, presence of scoliosis or thoracic hyper kyphosis, history of any surgery, and history of any upper limb injury were the exclusion criteria for the study prior to participation.

All of the athletes and or their guardian were informed about the study, and the written and verbal consent of the athletes and or their guardian was obtained. Ethical approval was obtained from a University Ethical Committee (2019/4/4), and the study was conducted according to the Helsinki Declaration (2008) Principles.

All the procedures of the study were completed within two consecutive days. On the first day, demographic data of the athletes was recorded, scapular asymmetry evaluation of the athletes was performed, sense of proprioception of the shoulder was evaluated, and shoulder horizontal abduction/adduction isokinetic muscle strength was measured. On the second day, shoulder internal and external rotation flexibility assessment was done, then shoulder abduction/adduction isometric muscle strength measurement was performed. Tests were conducted in the off-season period. The athletes were told not to train at least one day before the test. Athletes participated in the test rested.

\section{Evaluation of Scapular Asymmetry}

Kibler's "Lateral Scapular Slide Test" (LSST) was used to evaluate scapular asymmetry [13] by a physiotherapist. LSST was used to determine the position of the scapula at the 0,45 , and 90 degrees abduction position of the upper limb. LSST was performed bilaterally at three different positions. First with the arms in the neutral position at both sides. Secondly with shoulders at the $45^{\circ}$ abduction, the hands at the waist, and the thumbs turned to the back. Thirdly with shoulders at $90^{\circ}$ abduction, maximal internal rotated $[13,17]$.

Scapular position measurements were performed bilaterally in the three test positions by measuring the distance between the lower angle of the scapula and the spinous processes of the thoracic vertebrae. A $1 \mathrm{~cm}$ difference between the bilateral scapula in the scapular positions was the original level of a positive LSST. Later on, this level has been determined at a $1.5 \mathrm{~cm}$ difference to be accepted as a positive LSST [16]. According to the Kibler test, at least at one of three positions, athletes with at least $1.5 \mathrm{~cm}$ difference from the opposite side in the left side scapula (non-dominant side) in the scapular position measurements were included in the study group. Athletes with the same demographic characteristics, the same sports branch as the study group, and with neutral scapular position (less than $1 \mathrm{~cm}$ difference in every three tests according to the Kibler test) were included in the control group.

Demographic characteristics and professional experience characteristics of the athletes with and without scapular asymmetry was determined to be simi$\operatorname{lar}(p>0.05)$. It was determined that the average age of the study and control group, respectively was $15.63 \pm 2.17,15.89 \pm 1.76$ years; the average bodyweight of the study and control group, respectively was $56.44 \pm 12.94,54.16 \pm 10.23 \mathrm{~kg}$; the average height of the study and control group, respectively was $1.64 \pm 0.10,1.64 \pm 0.11 \mathrm{~m}$; the average Body Mass Index (BMI) of the study and control group, respectively were $20.82 \pm 3.14,19.86 \pm 2.17 \mathrm{~kg} / \mathrm{m}^{2}$; and the average professional experience of the study and control group, respectively was $7.71 \pm 3.11,7.05 \pm 2.91$ (Tab. 1).

\section{Muscle Strength Evaluation}

Shoulder horizontal abductor/adductor isokinetic muscle strength and shoulder abductor/adductor isometric muscle strength was evaluated with a IsoMed 2000 (D. \& R. Ferstl GmbH, Hemau, Germany) device. Before conducting both strength measurements, a standardized warm up with a reciprocal bicycle armleg ergometer at $60-70 \mathrm{rpm}$ for 10 minutes was performed by the athletes.

The isokinetic muscle strength of the horizontal abduction/adduction of the shoulder was examined with a dynamometer (IsoMed 2000, D. \& R. Ferstl GmbH, Hemau, Germany) as described in the user manual of the device when the athlete was in supine position. The strength of the dominant and non-dominant side shoulder horizontal abduction/adduction muscles was evaluated with five horizontal abduction/adduction exercises at $60^{\circ} / \mathrm{sec}$ angular velocity. Before the evaluation, three horizontal abduction/adduction exercises were performed to warm up and to practice. Firstly, the dominant right side was evaluated and 3 minutes rest period was given between the dominant and the non-dominant side. As a result of the evaluation; Peak Torque (PT) values were recorded for shoulder horizontal abduction/ adduction muscles and used in statistical analysis.

The isometric muscle strength of the abduction/adduction of the shoulder was examined with the shoulder module of the IsoMed 2000 device as described in the user manual of the device when the athlete was in a sitting position. The strength of the dominant and nondominant side shoulder abduction/adduction muscles was evaluated with ten-second isometric abduction/adduction exercises and this was done three times. Before the evaluation, one abduction/adduction exercise was performed to warm up and to practice. Firstly, the dominant right side was evaluated after 3 minutes, and then the non-dominant left side was evaluated. As a result of 
the evaluation; Peak Torque (PT) values were recorded for shoulder abduction/adduction muscles and the average of three PT values used in statistical analysis.

\section{Sense of Proprioception Evaluation}

A Digital Inclinometer (Dualer IQ Pro, Jtech Medical Industries, New York, USA) was used to evaluate the sense of proprioception of both shoulder joints. Sense of proprioception of the shoulder joint abduction was performed at 60,90, and 120 degrees abduction with three repetitions at each angle with Active Re-position Test. The athletes were seated on a chair without armrests with their feet in full contact with the ground and 90 degrees knee flexion. The eyes of the athletes were closed and secured with tape. The digital inclinometer was placed on the insertion of the deltoid muscle. The arm of the athlete was moved from the start position ( 0 degrees) to the target angles $(60,90,120$ degrees abduction) by the physiotherapist who performed the test. The arm of the athlete was kept at each target angle for 5 seconds to learn the position and then moved back to the start position. Next, the athletes were asked to move their arm six times to each target angle, and the difference between the target angle and actively moved angle was recorded. The average of the difference determined by six tests at each angle was then calculated and recorded, with these results used in the statistical analysis. The increased difference indicates a worse sense of proprioception.

\section{Evaluation of Shoulder Internal/External Rotation Flexibility}

The flexibility of shoulder internal/external rotation movements was evaluated by tape measure. The athletes were asked to slide their hands over their spine, and guided by their thumb. The thoracic 5th vertebral spinous process was accepted as a reference point to evaluate internal rotation, and the cervical 7 th vertebral spinous process was accepted as a reference point to evaluate external rotation. In order to evaluate internal rotation, the distance between the spinous process of the thoracic 5th vertebra and the thumb was measured. In order to evaluate external rotation, the distance between the spinous process of the cervical 7 th vertebra and the thumb was measured. In this measurement, the distance between the thumb and reference point was recorded as a positive value in the athletes passing the reference point, while it was recorded a negative value in the athletes who could not reach the reference point. For statistical analysis, the value of the athlete who reached the farthest from the reference point was accepted as the zero points, and the values determined according to the new zero points were used in the statistical analysis. It has been stated that the reliability of the maximum internal rotation flexibility measurement is 0.44 , and the reliability of the maximum external rotation flexibility measurement is 0.39 , and both measurements are reliable $[18,19]$.

\section{Statistical Analysis}

The SPSS for Windows 20.0 (Statistical Package for Social Sciences Inc. Chicago, IL, USA) package program was used for the statistical analysis of the study. Descriptive statistics of all variables were made and variables were shown as Mean \pm Standard Deviation $(\mathrm{X} \pm \mathrm{SS})$. The normal distribution of the data was determined by the Shapiro-Wilk test. Independent Samples T-test or Mann Whitney-U test and was used in the comparison of the data between the groups according to the normal distribution status. The effect size was determined by Cohen's d test. Cohen's $d=0.2,0.5$, and 0.8 , often is cited as indicative of a small, medium, and large effect size, respectively and that the corresponding values for $r$ are $0.1,0.3$, and 0.5 [20]. In the analysis of the data, the statistical significance level was set as $\mathrm{p}<0.05$.

\section{Results}

It was determined that there was no difference between the groups in dominant and non-dominant sides for shoulder horizontal abductor/adductor isokinetic muscle strength and strength balance at $60 \% / \mathrm{sec}$ angular velocity $(\mathrm{p}>0.05)$. There was no difference in isometric muscle strength of shoulder abductor/adductor muscles between the asymmetry and control group $(\mathrm{p}>0.05)$. There was no difference in sense of proprioception at $60^{\circ}, 90^{\circ}, 120^{\circ}$ shoulder abduction between the asymmetry and control group $(p>0.05)$. As a result of comparing the flexibility of the shoulder internal/external rotator muscles of the athletes with and without scapular asymmetry, it was determined that the group with asymmetry was more flexible in both the dominant and non-dominant side internal rotation $(\mathrm{p}<0.05$, z/t:2.369/-2.607, r/Cohen's D: $-0.447 / 0.845, \mathrm{df}=36$ ), and there was no difference between the flexibility of external rotation $(p>0.05)$ (Tab. 2).

\section{Discussion}

As a result of the study, there was no difference in the shoulder horizontal abduction-adduction isokinetic muscle strength, abduction-adduction isometric muscle strength, sense of proprioception, nor shoulder external rotation flexibility between adolescent athletes with and without scapular asymmetry. However, the 
Tab. 2. Comparison of isokinetic and isometric muscle strength, proprioception sense and flexibility of the groups

\begin{tabular}{|c|c|c|c|c|c|c|}
\hline & Isokinetic Muscle Strength & $\begin{array}{l}\text { Control Group } \\
\qquad(\mathrm{n}=19)\end{array}$ & $\begin{array}{l}\text { Asymmetry Group } \\
(\mathrm{n}=19)\end{array}$ & 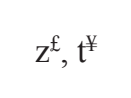 & $\mathrm{p}$ & Cohen's D/r \\
\hline \multirow{8}{*}{$\begin{array}{l}60^{\circ} / \mathrm{sec} \text { Angular } \\
\text { Velocity }\end{array}$} & DM ABD PT (Nm) & $50.57 \pm 16.56$ & $49.54 \pm 16.74$ & $-0.365^{\mathfrak{f}}$ & $0.715^{\mathfrak{f}}$ & 0.031 \\
\hline & ND ABD PT (Nm) & $58.81 \pm 13.37$ & $55.39 \pm 15.00$ & $-0.818^{£}$ & $0.414^{\mathfrak{1}}$ & 0.106 \\
\hline & DM ADD PT (Nm) & $53.61 \pm 18.87$ & $50.62 \pm 18.85$ & $-0.540^{£}$ & $0.589^{£}$ & 0.083 \\
\hline & ND ADD PT (Nm) & $50.57 \pm 17.95$ & $45.49 \pm 17.40$ & $0.886^{¥}$ & $0.382^{¥}$ & $0.287^{*}$ \\
\hline & DM ADD/ABD RATIO (\%) & $95.72 \pm 13.10$ & $100.98 \pm 21.51$ & $-0.511^{\mathfrak{f}}$ & $0.609^{\mathfrak{E}}$ & -0.141 \\
\hline & ND ADD/ABD RATIO (\%) & $123.43 \pm 30.31$ & $128.49 \pm 30.10$ & $-0.978^{\mathfrak{E}}$ & $0.328^{\mathfrak{E}}$ & -0.083 \\
\hline & ABD SYMMERY INDEX (\%) & $85.15 \pm 13.57$ & $89.36 \pm 18.35$ & $-0.423^{£}$ & $0.672^{\mathfrak{E}}$ & -0.126 \\
\hline & ADD SYMMERY INDEX (\%) & $108.25 \pm 18.98$ & $113.50 \pm 22.28$ & $-1.095^{\mathfrak{f}}$ & $0.274^{\mathfrak{f}}$ & -0.123 \\
\hline \multirow{4}{*}{$\begin{array}{l}\text { Isometric } \\
\text { Muscle } \\
\text { Strength }\end{array}$} & Dominant Abduction $(\mathrm{N})$ & $47.01 \pm 22.42$ & $47.01 \pm 20.52$ & $-0.307^{\mathfrak{1}}$ & $0.759^{£}$ & 0 \\
\hline & Non-Dominant Abduction (N) & $47.69 \pm 22.20$ & $50.60 \pm 30.42$ & $-0.161^{£}$ & $0.872^{\mathfrak{E}}$ & -0.056 \\
\hline & Dominant Adduction (N) & $55.97 \pm 23.19$ & $62.47 \pm 30.98$ & $-0.497^{\mathfrak{f}}$ & $0.619^{\mathfrak{E}}$ & -0.129 \\
\hline & Non-Dominant Adduction (N) & $53.87 \pm 22.34$ & $60.68 \pm 31.50$ & $-0.769^{¥}$ & $0.447^{¥}$ & $0.249^{*}$ \\
\hline \multirow{6}{*}{$\begin{array}{l}\text { Proprioception } \\
\text { Sense }\end{array}$} & $60^{\circ}$ Dominant Error $\left(^{\circ}\right)$ & $5.15 \pm 3.03$ & $4.22 \pm 4.34$ & $-1.621^{\mathfrak{E}}$ & $0.105^{\mathfrak{f}}$ & 0.140 \\
\hline & $60^{\circ}$ Non-Dominant Error $\left(^{\circ}\right)$ & $5.17 \pm 4.49$ & $6.04 \pm 3.28$ & $-0.876^{£}$ & $0.381^{\mathfrak{f}}$ & -0.140 \\
\hline & $90^{\circ}$ Dominant Error $\left(^{\circ}\right)$ & $4.30 \pm 2.81$ & $5.00 \pm 3.32$ & $-0.964^{£}$ & $0.335^{\mathfrak{f}}$ & -0.192 \\
\hline & $90^{\circ}$ Non-Dominant Error $\left(^{\circ}\right)$ & $3.96 \pm 2.80$ & $4.20 \pm 2.60$ & $-0.599^{£}$ & $0.549^{\mathfrak{E}}$ & -0.242 \\
\hline & $120^{\circ}$ Dominant Error $\left(^{\circ}\right)$ & $5.61 \pm 4.34$ & $5.33 \pm 3.38$ & $-0.234^{\mathfrak{f}}$ & $0.815^{\mathfrak{f}}$ & 0 \\
\hline & $120^{\circ}$ Non-Dominant Error $\left(^{\circ}\right)$ & $5.46 \pm 3.86$ & $4.24 \pm 2.20$ & $-0.628^{£}$ & $0.530^{\mathfrak{f}}$ & 0.192 \\
\hline \multirow{4}{*}{ Flexibility } & Thumb T5 DM (cm) & $8.55 \pm 4.35$ & $12.38 \pm 4.28$ & $-2.369^{£}$ & $0.018^{£}$ & -0.447 \\
\hline & Thumb T5 ND (cm) & $10.97 \pm 4.53$ & $14.28 \pm 3.18$ & $-2.607^{¥}$ & $0.013^{¥}$ & $0.845^{*}$ \\
\hline & Thumb C7 DM (cm) & $6.38 \pm 2.98$ & $5.24 \pm 2.51$ & $-0.891^{£}$ & $0.373^{\mathfrak{E}}$ & 0.242 \\
\hline & Thumb C7 ND (cm) & $5.92 \pm 2.79$ & $5.46 \pm 2.14$ & $-0.526^{£}$ & $0.559^{£}$ & 0 \\
\hline
\end{tabular}

p: comparison of study and control group $\left({ }^{£}\right.$ Mann Whitney U Test, ${ }^{\ddagger}$ Independent Samples T Test), Effect size: *Cohen’s D; others r, DM: Dominant side, NDM: Non-Dominant side, ABD: Horizontal abduction, ADD: Horizontal adduction, PT: Peak Torque, Nm: Newton Meter, N: Newton.

shoulder internal rotation flexibility was higher in athletes with scapular asymmetry than the athletes without asymmetry.

There are various studies reporting that individuals with scapular asymmetry have a loss of strength in the shoulder girdle muscles [21-23]. It is shown reduced infraspinatus and supraspinatus muscle strength in the symptomatic overhead athletes with scapular asymmetry [22]. Merolla et al., suggested that the imbalances in capsular muscle systems can cause infraspinatus and supraspinatus muscles weakness, and this weakness might be a reason for scapular asymmetry by changing the length-tension relationship of the rotator cuff muscles $[22,23]$. Likewise, Hannah et al. examined healthy people with scapular asymmetry; reduced muscle strength was reported in the upper, middle, and lower part of the trapezius muscle, as well as in the anterior muscle of the serratus [24]. In the current study; there was no difference between the athletes with and without scapular asymmetry in the horizontal abduction nor adduction muscle strength. This study showed the shoulder horizontal abductor muscles which pull the scapula to the medial line, and shoulder horizontal adductor muscles, which are the antagonist of the horizontal abductors of the asymmetric athletes are strong as symmetric athletes. There is 
not any study examine these muscles and this can be the reason why the results of the study are different from the literature.

It is stated that there is a relationship between scapular asymmetry and shoulder injuries [3-6,11,12,25]. Therefore, considering the biomechanical disorder caused by scapular asymmetry may have not only a scapulothoracic effect but also a scapulohumeral effect $[3-6,11,12,25]$. When we evaluated shoulder abductor/ adductor muscle strength in our study. This study, the isometric muscle strength of the shoulder abductor/adductor was similar between the two groups. Evaluating both isokinetic horizontal abduction/adduction and isometric abduction/adduction muscle strength of the shoulder, and including only elite athletes to this study differentiates from the current study in the literature.

Scapular asymmetry lead to biomechanical abnormalities and change periscapular muscle activity [8]. This can affect the position of joint and proprioception. Proprioception disorders were shown in individuals with shoulder pathology such as shoulder impingement syndrome, shoulder instability, and anterior glenohumeral dislocation history [10]. The shoulder sense of proprioception was compared between 61 patients with subacromial impingement and 30 healthy participants. The sense of proprioception was found to be worse in patients with impingement syndrome [9]. As a result of our study, the sense of proprioception of the shoulder was similar between the groups. It is thought that participation of the athletes without a shoulder injury, a stronger association of scapular asymmetry with scapular asymmetry scapulothoracic joint than the glenohumeral joint might be the reason for this result. It is suggested that the sense of proprioception of the scapulothoracic joint should be evaluated as the sense of proprioception of the glenohumeral joint, in the presence of the scapular asymmetry [26].

Pectoralis minor muscle length affects the scapular kinematics in both asymptomatic and symptomatic individuals, and the pectoralis minor muscle is found to be shorter in the case of scapular asymmetry $[27,28]$. The pectoralis minor muscle is an anatomical structure directly attached to the scapula; therefore, the reduced length of this muscle is expected to affect the scapular position or orientation $[27,28]$. In our study, both dominant and nondominant shoulder internal rotation flexibility of asymmetric group athletes were found to be higher than the control group. The possible reason for this is thought to be related to the pectoralis minor muscle length $[27,28]$. In our study, we did not evaluate the length of the pectoralis minor muscle. Therefore, studies to examine muscle lengths such as soft tissue ultrasonography in the case of scapular asymmetry are needed to demonstrate this situation. Scapular asymmetry is evaluated with the distance between the medial angle of the scapula and the midline of the trunk. In our study, the flexibility of both dominant and nondominant shoulders in the asymmetric group was higher than the control group's internal rotation flexibility. This result indicates that the asymmetry might be bilateral in the asymmetry group. Bilateral asymmetry might be investigated with three-dimensional motion analysis. In addition, it is stated in the literature that there is a relationship between internal rotation and shoulder injuries, and the possibility of shoulder injury increases as the internal rotation flexibility increases [14]. Increased internal rotation flexibility of the scapular asymmetry group may indicate the risk of shoulder injuries. However, this idea should be examined with future longterm follow-up studies.

The participation of the asymptomatic athletes, the evaluation of the scapular asymmetry with LSST, the relation of measured parameters with the glenohumeral joint, not normally distributed data, relatively small sample size and mix of sports included in this study could be accepted as limitations of our study. Evaluation of both scapulohumeral and scapulothoracic muscle strength with an isokinetic device, while designing the study as a matched control study are the strengths of our study.

\section{Conclusion}

As a result of our study, there was no difference in the shoulder horizontal abduction-adduction isokinetic muscle strength, abduction-adduction isometric muscle strength, sense of proprioception, nor shoulder external rotation flexibility between adolescent athletes with and without scapular asymmetry. It was determined that the flexibility of shoulder internal rotation in adolescent athletes with scapular asymmetry was higher than those without asymmetry.

\section{Funding}

This research received no external funding.

\section{Conflicts of interest}

The authors declare no conflict of interest.

\section{References}

1. Ben Kibler W. The role of the scapula in athletic shoulder function. Am J Sports Med. 1998; 26(2): 325-37.

2. Wilk KE, Arrigo C. Current concepts in the rehabilitation of the athletic shoulder. J Orthop Sports Phys Ther. 1993; 18(1): 365-78. 
3. Kibler W. Biomechanical analysis of the shoulder during tennis activities. Clin Sports Med. 1995; 14(1): 79-85.

4. Phadke V, Camargo P, Ludewig P. Scapular and rotator cuff muscle activity during arm elevation: a review of normal function and alterations with shoulder impingement. Braz J Phys Ther. 2009; 13(1): 1-9.

5. Kibler BW, Sciascia A, Wilkes T. Scapular dyskinesis and its relation to shoulder injury. J Am Acad Orthop Surg. 2012; 20(6): 364-72.

6. Gumina S, Carbone S, Postacchini F. Scapular dyskinesis and SICK scapula syndrome in patients with chronic type III acromioclavicular dislocation. Arthroscopy. 2009; 25(1): 40-5.

7. Turgut E, Duzgun I, Baltaci G. Scapular asymmetry in participants with and without shoulder impingement syndrome; a three-dimensional motion analysis. Clin Biomech. 2016; 39: 1-8.

8. Karagiannakis D, Athanasopoulos S, Mandalidis D. Scapular muscles' activity in female volleyball players with scapular asymmetry in the resting position. J Bodyw Mov Ther. 2018; 22(3): 580-5.

9. Sahin E, Dilek B, Baydar M, Gundogdu M, Ergin B, Manisali M, et al. Shoulder proprioception in patients with subacromial impingement syndrome. J Back Musculoskelet Rehabil. 2017; 30(4): 857-62.

10. Kawasaki T, Yamakawa J, Kaketa T, Kobayashi H, Kaneko K. Does scapular dyskinesis affect top rugby players during a game season? J Shoulder Elbow Surg. 2012; 21(6): 709-14.

11. Oyama S, Myers JB, Wassinger CA, Daniel Ricci R, Lephart SM. Asymmetric resting scapular posture in healthy overhead athletes. J Athl Train. 2008; 43(6): 565-70.

12. Ludewig PM, Reynolds JF. The association of scapular kinematics and glenohumeral joint pathologies. J Orthop Sports Phys Ther. 2009; 39(2): 90-104.

13. Kim S-R, Kang M-H, Bahng S-Y, An J-K, Lee J-Y, Park S-Y, et al. Correlation among scapular asymmetry, neck pain, and neck disability index (NDI) in young women with slight neck pain. J Phys Ther Sci. 2016; 28(5): 1508-10.

14. Shih Y-F, Wang Y-C. Spiking Kinematics in Volleyball Players with Shoulder Pain. J Athl Train. 2019; 54(1): 90-8.

15. Lin Y-L, Karduna A. Exercises focusing on rotator cuff and scapular muscles do not improve shoulder joint position sense in healthy subjects. Hum Mov Sci. 2016; 49: 248-57.

16. Curtis T, Roush JR. The lateral scapular slide test: A reliability study of males with and without shoulder pathology. N Am J Sports Phys Ther. 2006; 1(3): 140-6.
17. Odom CJ, Taylor AB, Hurd CE, Denegar CR. Measurement of scapular asymmetry and assessment of shoulder dysfunction using the lateral scapular slide test: a reliability and validity study. Phys Ther. 2001; 81(2): 799-809.

18. Edwards TB, Bostick RD, Greene CC, Baratta RV, Drez D. Interobserver and intraobserver reliability of the measurement of shoulder internal rotation by vertebral level. J Shoulder Elbow Surg. 2002; 11(1): 40-2.

19. Hayes K, Walton JR, Szomor ZL, Murrell G. Reliability of five methods for assessing shoulder range of motion. Aust J Physiother. 2001; 47(4): 289-96.

20. Rice, M.E. and G.T. Harris. Comparing effect sizes in follow-up studies: ROC Area, Cohen's d, and r. Law Hum Behav. 2005; 29(5): 615-20.

21. Seitz AL, McClelland RI, Jones WJ, Jean RA, Kardouni JR. A comparison of change in 3D scapular kinematics with maximal contractions and force production with scapular muscle tests between asymptomatic overhead athletes with and without scapular dyskinesis. Int J Sports Phys Ther. 2015; 10(3): 309-18.

22. Merolla G, De Santis E, Campi F, Paladini P, Porcellini G. Supraspinatus and infraspinatus weakness in overhead athletes with scapular dyskinesis: strength assessment before and after restoration of scapular musculature balance. Musculoskelet Surg. 2010; 94(3): 119-25.

23. Merolla G, De Santis E, Sperling JW, Campi F, Paladini P, Porcellini G. Infraspinatus strength assessment before and after scapular muscles rehabilitation in professional volleyball players with scapular dyskinesis. J Shoulder Elbow Surg. 2010; 19(8): 1256-64.

24. Hannah DC, Scibek JS, Carcia CR. Strength profiles in healthy individuals with and without scapular dyskinesis. Int J Sports Phys Ther. 2017; 12(3): 305-13.

25. McClure $P$, Tate AR, Kareha S, Irwin D, Zlupko E. A clinical method for identifying scapular dyskinesis, part 1: reliability. J Athl Train. 2009; 44(2): 160-4.

26. Lin Y-L, Karduna A. Errors in shoulder joint position sense mainly come from the glenohumeral joint. J Appl Biomech. 2017; 33(1): 32-8.

27. Borstad JD, Ludewig PM. The effect of long versus short pectoralis minor resting length on scapular kinematics in healthy individuals. J Orthop Sports Phys Ther. 2005; 35(4): 227-38.

28. Borich MR, Bright JM, Lorello DJ, Cieminski CJ, Buisman T, Ludewig PM. Scapular angular positioning at end range internal rotation in cases of glenohumeral internal rotation deficit. J Orthop Sports Phys Ther. 2006; 36(12): 926-34. 